\title{
Utilização do malte de sorgo na produção de cerveja: revisão bibliográfica
}

\author{
Use of sorghum malt in brewing: review
}

\author{
Yanet Boffill Rodríguez" ${ }^{1 *}$, Irenia Gallardo Aguilar², João Batista de Almeida e Silva' \\ ${ }^{1}$ Universidade de São Paulo (USP), Escola de Engenharia de Lorena (EEL), Departamento de Biotecnologia, Lorena/SP - Brasil \\ 2 Universidade Central "Marta Abreu" de Las Villas, Departamento de Engenharia Química, Villa Clara/Santa Clara - Cuba
}

\section{${ }^{*}$ Corresponding Author}

Yanet Boffill Rodríguez, Universidade de São Paulo (USP), Escola de Engenharia de Lorena (EEL), Departamento de Biotecnologia, Rodovia Itajuba-Lorena, km 74,5, CEP: 12602-810, Lorena/SP - Brasil, e-mail: yanetb@usp.br

Cite as: Use of sorghum malt in brewing: review. Braz. J. Food Technol., v. 21, e2017198, 2018.

Received: Mar. 14, 2017; Approved: June 21, 2018

\section{Resumo}

A cevada (Hordeum vulgare) é o principal cereal utilizado na produção de cerveja, mas é importada, na maioria dos países tropicais e subtropicais. Isto implica no aumento do custo de produção da bebida, além de não satisfazer a demanda das bebidas sem glúten no mercado. O sorgo (Sorghum bicolor L. Moench) é um dos cereais que também podem ser usados na produção de cerveja e, ao contrário da cevada, é livre de glúten, recomendado para doentes celíacos. Assim, recentemente tem aumentado o interesse no uso do malte de sorgo para substituir o malte de cevada na produção de bebidas. No entanto, as principais dificuldades encontradas na produção de bebidas fermentadas a partir de malte de sorgo têm sido o baixo nível de enzimas hidrolíticas e a alta temperatura de gelatinização. O objetivo deste trabalho de revisão foi avaliar os principais fatores que afetam os processos de maltagem (tempo e temperatura de imersão, de germinação e de secagem) e a mosturação com malte de sorgo ( $\mathrm{pH}$ e temperatura das enzimas, e métodos usados) na produção de cerveja sem glúten.

Palavras-chave: Bebidas; Cereais; Cevada; Enzimas; Maltagem; Mosturação; Sem glúten; Substituição.

\section{Abstract}

Barley (Hordeum vulgare) is the main cereal used in beer production, but is imported in most of the tropical and subtropical countries. This increases the production costs, without satisfying the market demand for gluten-free beverages. Sorghum (Sorghum bicolor L. Moench) is one of the cereals that can also be used in brewing, and, unlike barley, is gluten-free and hence recommended for celiac patients. Thus interest in the use of sorghum malt to replace barley malt in beverage production has increased recently. However, the main difficulties encountered in the production of fermented beverages using sorghum malt have been the low level of hydrolytic enzymes and high gelatinization temperature. Hence the objective of this review was to evaluate the main factors affecting malting processes (drying and germination, steeping time and temperature) and mashing with sorghum malt ( $\mathrm{pH}$ and temperature of the enzymes and methods used) in gluten-free beer production.

Keywords: Beverage; Cereals; Barley; Enzymes; Malting; Mashing; Gluten-free; Replacement.

\section{Introdução}

Apesar das numerosas vantagens da cevada sobre outros cereais, no processo de produção de cerveja, tem aumentado o interesse pelas pesquisas sobre o uso de cereais tropicais para oferecer bebidas sem glúten, que possam ser consumidas pelos doentes celíacos, e que possibilitem também reduzir os custos de produção (ARCHIBONG et al., 2015; ABUAJAH et al., 2016a). Conforme Fasano e Catassi
(2001), a doença celíaca (CD), em indivíduos geneticamente suscetíveis, é uma síndrome caracterizada por dano da mucosa do intestino delgado, causada pela fração de gliadina de glúten do trigo e de proteínas semelhantes (prolaminas) da cevada e do centeio. Os autores afirmam que o tratamento chave da CD é a dieta, ao longo da vida, na qual os produtos que contêm glúten são eliminados. Nesse sentido, há vários 
estudos, direcionados aos doentes celíacos, sobre alimentos livres de glúten, tais como bolos (GOMES et al., 2014), biscoitos (MARIANI et al., 2015) e pães (SILVA et al., 2017). É assumido que o sorgo tem grande potencial para ser usado na produção de alimentos e bebidas sem glúten (ARENDT; SCHNITZENBAUMER, 2014). O sorgo pertence à família Graminae e ao gênero sorghum (OWUAMA, 1997). O sorgo, ao contrário da cevada, é muito bem adaptado às condições semiáridas e subtropicais. Uma das vantagens de seu cultivo é que ele pode crescer sob forte estresse ambiental, como a seca, em que os cereais temperados não conseguem se desenvolver (AGU; PALMER, 1998). Em países da América, o sorgo tem um preço relativamente mais baixo, quando comparado ao da cevada. Por exemplo, os preços registrados do sorgo (USD/ton) e da cevada para Bolívia, Colômbia, México, Uruguai e EUA têm sido 163,50 e 407,30, 300,20 e 429,10, 189,20 e 235,70, 156, 10 e 227,30, 120,00 e 253,00, respectivamente (FAO, 2015). Contudo, as principais dificuldades encontradas no uso do sorgo, na produção cervejas, são a alta temperatura de gelatinização $\left(>70^{\circ} \mathrm{C}\right)$ e o baixo poder diastático (DP) (OWUAMA, 1997; OGBONNA, 2011). Portanto, o foco das pesquisas tem sido melhorar a qualidade do malte (DJAMEH et al., 2015; EBBAH et al., 2015). Visando usar o sorgo na produção de cervejas, para aproveitar suas potencialidades, é necessário o conhecimento dos fatores que influenciam na qualidade de seu malte e na mosturação; assim, tais informações podem ajudar a identificar o impacto sobre o processo geral. Para viabilizar a etapa de fermentação, é necessário conhecer, além do tipo de cultivar, as faixas adequadas de variáveis independentes, tais como: i) na maltagem: tempo e temperatura de imersão, de germinação e de secagem; ii) na mosturação: pH e temperatura das enzimas, e métodos. Dessa forma, é possível usar as informações adquiridas com a finalidade de produzir bebidas sem glúten, com baixo teor alcoólico. Portanto, nesta revisão, buscou-se reunir os estudos que levam a aceitáveis parâmetros de qualidade, tais como perdas da maltagem (MWL), poder diastático, extrato em água fria (CWE), extrato em água quente (HWE), atenuação limite (AAL), amino nitrogênio livre (FAN), nitrogênio solúvel total (TSN) e Índice de Kolbach (KI), de modo que tais informações possam auxiliar na avaliação do potencial de variedades não estudadas para a produção de bebidas fermentadas sem glúten.

\subsection{Limitações do sorgo na produção de cerveja}

Conforme Owuama e Okafor (1987), o processo de produção de cerveja consta de seis etapas principais: maltagem, mosturação, fervura do mosto, fermentação, maturação e engarrafamento. Contudo, esta revisão mostra que as limitações do processo, a partir do malte de sorgo, estão relacionadas, principalmente, com as etapas de maltagem e de mosturação. Além da alta temperatura de gelatinização, o malte de sorgo apresenta insuficiente nível de enzimas hidrolíticas, quando comparado com o malte da cevada, especificamente o nível de $\beta$-amilase. Taylor e Robbins (1993) relataram que os grãos de sorgo não germinados não exibem atividade $\beta$-amilase e que, depois da maltagem, o sorgo tem uma atividade $\beta$-amilase inferior aos $25 \%$ do nível dessa atividade no malte de cevada. Por outro lado, tem sido reportado que o tempo de germinação é um fator crítico na atividade $\beta$-amilase (HASSANI et al., 2014). As causas da baixa atividade $\beta$-amilase têm sido explicadas por vários autores. Por exemplo, Uriyo e Eigel (1999) explicaram que essa debilidade é devida à interação entre a $\beta$-amilase e polifenóis. Diversamente, Dufour et al. (1992) mostraram que a atividade $\beta$-amilase permanece fraca, mesmo em variedades de sorgo com baixo teor de polifenóis. Os autores atribuíram os problemas de sacarificação, nas variedades de sorgo estudadas, à grande variabilidade da temperatura de gelatinização e viscosidade do amido, e à falta de $\beta$-amilase em muitos dos grãos. A variabilidade no comportamento da temperatura de gelatinização e das viscosidades foi atribuída a porcentagens variáveis de ramificação em amilopectina e diferentes forças de ligação nos grânulos (maior resistência ao inchamento e à ruptura). Outros autores afirmam que, à parte dos polifenóis, o baixo nível de proteínas solúveis também pode inibir a atividade $\beta$-amilase. As proteínas formam uma matriz que envolve os grânulos de amido e, se não são solubilizadas durante a germinação, inibem a hidrólise do amido pela $\beta$-amilase (PALMER et al., 1989). Por outra parte, a proteólise é vital para a geração de FAN e peptídeos curtos, durante a maltagem. No sorgo, a atividade proteolítica também é outra limitação. Segundo Evans e Taylor (1990), a proteólise é provocada pela ação das enzimas proteolíticas do malte. O baixo grau de proteólise (atividade proteolítica) afeta negativamente o rendimento, a filtração e a fermentabilidade do mosto, como também a espuma e a estabilidade da cerveja. Um grau de proteólise adequado é decorrente de adequados níveis de TSN e de FAN. Além disso, a degradação incompleta das proteínas estruturais e de armazenamento do grão, durante a maltagem, limita a extensão da solubilização de carboidratos, durante a mosturação (AGU; PALMER, 1997). Como observado, os principais obstáculos do malte de sorgo para ser usado na produção de cerveja são decorrentes das ações limitadas das enzimas amilolíticas e proteolíticas; dessa maneira, fica comprometida a produção de malte de boa qualidade devido à baixa atividade enzimática. Isso proporciona as desvantagens econômicas relacionadas com a filtração do mosto. Entretanto, essas limitações podem ser minimizadas por meio de: (i) a escolha adequada da variedade de sorgo, que apresente baixa temperatura de gelatinização e alto poder diastático; (ii) otimização da maltagem, e (iii) otimização da mosturação. 


\subsection{Maltagem}

Agu e Palmer (1996) destacaram que o objetivo principal do processo da maltagem é gerar as enzimas hidrolíticas, as quais clivam os compostos principais, amido e proteínas, para proporcionar açúcares fermentáveis, nitrogênio amino livre e aminoácidos. As três etapas que

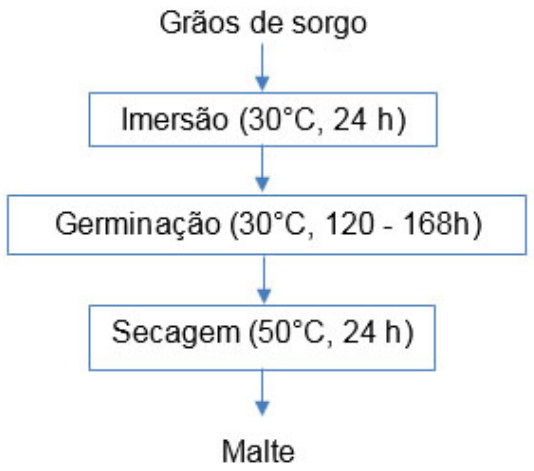

Figura 1. Fluxograma do processo de maltagem de sorgo. Fonte: do autor. envolvem este processo, assinaladas por Dewar et al. (1997), assim como as melhores condições na maltagem de sorgo, extraídas desta revisão, são mostradas na Figura 1. Geralmente, a qualidade do malte de sorgo é influenciada pelas condições da maltagem, tais como tempo e temperatura de imersão, de germinação e de secagem, e pela variedade. Visando incentivar o desenvolvimento de pesquisas com variedades de sorgo diferentes às já estudadas, as Tabelas 1 e 2 mostram um resumo das condições do processo e das cultivares estudadas, das condições otimizadas e dos parâmetros de qualidade obtidos na maltagem, respectivamente.

\subsubsection{Parâmetros de qualidade do malte}

\subsubsection{Perdas da maltagem}

As perdas da maltagem são aquelas registradas como resultado de processos metabólicos associados à germinação dos grãos, que se manifestam pelo crescimento e desenvolvimento das raízes e gêmulas (AISEN; MUTS, 1987). A faixa de perda entre $10 \%$ e $15 \%$ é aceitável na

Tabela 1. Condições do processo de maltagem e cultivares de sorgo estudadas para a produção de cerveja sem glúten.

\begin{tabular}{|c|c|c|c|c|c|c|c|c|c|}
\hline Cultivar & $\begin{array}{l}\text { SD } \\
(\%)\end{array}$ & $\frac{\text { ST }}{\left({ }^{\circ} \mathrm{C}\right)}$ & St & $\frac{\mathrm{NaOH}}{(\%)}$ & $\frac{\mathrm{GT}}{\left({ }^{\circ} \mathbf{C}\right)}$ & $\frac{\text { Gt }}{\text { (h) }}$ & $\frac{\mathrm{DT}}{\left({ }^{\circ} \mathrm{C}\right)}$ & $\frac{\text { Dt }}{\text { (h) }}$ & Referência \\
\hline \multicolumn{10}{|c|}{ Estudos das condições da maltagem } \\
\hline ICSV 400, KSV 8 & $40-42$ & 30 & $48^{a}$ & - & 30 & 96 & 50 & 24 & $\begin{array}{c}\text { (EZEOGU; OKOLO } \\
1995)\end{array}$ \\
\hline KSV 13 & $34-35$ & 20 & $44^{a}$ & - & 20 & 120 & 50 & 16 & (AGU; PALMER, 1996) \\
\hline Bernard Red & 38 & $25-30$ & $40^{a}$ & - & 25 & 144 & 50 & 24 & (DEWAR et al., 1997) \\
\hline Kigufi & - & 25 & 24 & - & 30 & 72 & 50 & 24 & $\begin{array}{c}\text { (LYUMUGABE et al., } \\
\text { 2015) }\end{array}$ \\
\hline Kadaga & 40 & 40 & $14^{a}$ & 0.2 & 28 & 120 & 50 & 16 & (EBBAH et al., 2015) \\
\hline Sorgo amarelo & - & 25 & 8 & - & 30 & 96 & 50 & - & (NDUBISI et al., 2016) \\
\hline \multicolumn{10}{|c|}{ Estudos das cultivares } \\
\hline SK 5912, KSV 4, KSV 8, ICSV 400 & $33-35$ & 20 & $40^{a}$ & - & 30 & 120 & 50 & 16 & (OGU et al., 2006) \\
\hline NK 283, PAN 8586 & - & 25 & 8 & 0.2 & 28 & 144 & 50 & 24 & $\begin{array}{l}\text { (LEFYEDI; TAYLOR, } \\
\text { 2006) }\end{array}$ \\
\hline ICSV 400, ICSH 89009, ICSH 89002 & - & $30-40^{b}$ & $45^{\mathrm{a}}$ & - & 30 & $96-144$ & 50 & 24 & (OKOLI et al., 2010) \\
\hline $\begin{array}{c}\text { Gambella 1107, Macia, Meko, Red-Swazi, } \\
\text { Teshale, 76T1\#23 }\end{array}$ & - & 25 & $24^{a}$ & 0.2 & 28 & 96 & 50 & 24 & (BEKELE et al., 2012) \\
\hline $\begin{array}{c}\text { Phofu, Segaolane, Lekgeberwa, Town, } \\
\text { Sefofu, Kanye Standard, Marupantshi, } \\
\text { Mafia, Lars Vyt, BSH-1, SNK }\end{array}$ & - & 30 & 51 & - & 30 & - & 50 & 24 & $\begin{array}{l}\text { (MOKHAWA et al., } \\
\text { 2013) }\end{array}$ \\
\hline $\begin{array}{c}\text { SK 5912, KSV 8, ICSV 400, ICSV III, White } \\
\text { Kaura, Boboje, CSRO2, NRL-3, KAT 487, } \\
\text { Nafelen } 6\end{array}$ & - & 28 & 24 & - & - & 96 & 48 & 24 & (NNAMCHI et al., 2014) \\
\hline SAMSORG17, SAMSORG14, SAMSORG40 & $39-42$ & $28-30$ & $45^{\mathrm{a}}$ & - & $28-30$ & 120 & 50 & 16 & $\begin{array}{l}\text { (ABUAJAH et al., } \\
\text { 2016a) }\end{array}$ \\
\hline SK 5912, KSV 8, ICSV 400 & - & $28-30$ & $45^{\mathrm{a}}$ & - & $28-30$ & 120 & 50 & 16 & $\begin{array}{l}\text { (ABUAJAH et al., } \\
\text { 2016b) }\end{array}$ \\
\hline $\begin{array}{l}\text { Oito tipos de sorgo segundo as } \\
\text { características dos grãos. }\end{array}$ & - & 25 & $24^{a}$ & - & 25 & 72 & 50 & 24 & (MEZGEBE et al., 2018) \\
\hline Kpika, Dobi, Swan & $42-43$ & - & - & - & 27 & 72 & 50 & 24 & $\begin{array}{l}\text { (TOKPOHOZIN et al., } \\
\text { 2018) }\end{array}$ \\
\hline
\end{tabular}

SD: grau de imersão; ST: temperatura de imersão; St: tempo de imersão; $\mathrm{NaOH}$ : hidróxido de sódio; GT: temperatura de germinação; Gt: tempo de germinação; DT: temperatura de secagem; Dt: tempo de secagem; AR: repouso ao ar. a Uso de AR. 'blmersão final com água quente (40 $\left.{ }^{\circ} \mathrm{C}\right)$. 
Tabela 2. Condições ótimas e parâmetros de qualidade no processo de maltagem do sorgo.

\begin{tabular}{|c|c|c|c|c|c|c|c|c|c|}
\hline \multirow[b]{2}{*}{ Cultivar } & \multicolumn{7}{|c|}{ Condições ótimas } & \multirow[b]{2}{*}{ Parâmetros de qualidade } & \multirow[b]{2}{*}{ Referência } \\
\hline & $\frac{\text { SD }}{(\%)}$ & $\frac{\text { ST }}{\left({ }^{\circ} \mathrm{C}\right)}$ & St & $\begin{array}{c}\text { GT } \\
\left({ }^{\circ} \mathbf{C}\right)\end{array}$ & Gt & $\frac{\text { DT }}{\left({ }^{\circ} \mathbf{C}\right)}$ & Dt & & \\
\hline IS 2941 & - & 30 & 18 & 30 & 108 & 100 & $3-4^{b}$ & $\begin{array}{l}\text { MWL (\%): 19,5; CWE (\%): } \\
\text { 25,6; HWE (\%): 82,4; Açúcares } \\
\text { redutores no HWE }(\%): \\
\text { 81,1; DP }\left(\text { SDU g }{ }^{-1}\right): 58,9 ; \\
\text { LP }\left(g \text { amido g malte } \text { m }^{-1}\right): 60-70\end{array}$ & $\begin{array}{l}\text { (PATHIRANA et al., } \\
\text { 1983) }\end{array}$ \\
\hline $\begin{array}{c}\text { Sorgo } \\
\text { vermelho }\end{array}$ & - & 31 & 24 & 30 & 108 & - & - & $\begin{array}{l}\text { MWL (\%): 7,96; SSY (\%): } 31,8 ; \\
\text { Viscosidade do mosto (poise): } \\
\text { 17,26; Atividade amilase (unidade } \\
\text { de maltose): 7,95; Teor de taninos } \\
\text { (\%): 1,27; Proteínas (\%): } 31,73\end{array}$ & (CLAVER et al., 2010) \\
\hline- & 41 & 48 & - & 27 & 168 & 50 & 24 & $\begin{array}{c}\alpha \text {-amilase }\left(\mathrm{U} \mathrm{g}^{-1}\right): 139 ; \beta \text {-amilase } \\
\left(\mathrm{U} \mathrm{g}^{-1}\right): 60 ; \mathrm{HWE}(\%): 83,8 ; \mathrm{FAN} \\
(\mathrm{mg} / 100 \mathrm{~g}): 117,8 ; \mathrm{KI}(\%): 26,6\end{array}$ & $\begin{array}{l}\text { (HASSANI et al., } \\
\text { 2014) }\end{array}$ \\
\hline $\begin{array}{c}\text { Sorgo } \\
\text { vermelho }\end{array}$ & - & 25 & $32^{a}$ & 30 & 120 & 50 & 24 & $\begin{array}{c}\text { DP }\left({ }^{\circ} \mathrm{L}\right): 32 ; \operatorname{HWE}\left(\left({ }^{\circ} \mathrm{L} \mathrm{kg}^{-1}\right): 327\right. \\
\text { TSN }(\%): 0,45\end{array}$ & $\begin{array}{c}\text { (AGU; PALMER, } \\
\text { 2013) }\end{array}$ \\
\hline Chireh & - & 29 & $12-12.5^{a}$ & 30 & 120 & 40 & 16 & $\begin{array}{c}\text { DP }\left(\text { SDU g }{ }^{-1}\right): 42.17 ; \operatorname{HWE}(\%): \\
80,10 ; \text { AAL }(\%): 76,20 ; \text { FAN } \\
\left(\mathrm{mg} \mathrm{L}^{-1}\right):>100\end{array}$ & $\begin{array}{l}\text { (DJAMEH et al., } \\
\text { 2015) }\end{array}$ \\
\hline
\end{tabular}

SD: grau de imersão; ST: temperatura de imersão; St: tempo de imersão; GT: temperatura de germinação; Gt: tempo de germinação; DT: temperatura de secagem; Dt: tempo de secagem; MWL: perdas da maltagem; CWE: extrato em água fria; HWE: extrato em água quente; DP: poder diastático; SDU: unidades diastáticas de sorgo; LP: poder de liquefação; SSY: rendimento de sólidos solúveis; FAN: amino nitrogênio livre; KI: índice de Kolbach; TSN: nitrogênio solúvel total; AAL: atenuação limite; AR: repouso ao ar. aUso de AR. ${ }^{b}$ Antes de iniciar o tratamento, o malte foi seco a $45{ }^{\circ} \mathrm{C}$ até atingir uma umidade de $9,9 \%(\mathrm{p} / \mathrm{p})$.

maltagem de sorgo (NOVELLIE, 1962a). Geralmente, as perdas estão em proporção direta com o tempo de germinação e são maiores nos últimos dias de germinação (BEKELE et al., 2012; NNAMCHI et al., 2014). As altas perdas dos grãos de sorgo são atribuídas à temperatura de maltagem predominante nos cenários tropicais $\left(30^{\circ} \mathrm{C}\right)$, a qual é adequada para a germinação desse cereal (AGU; PALMER, 1998; OGU et al., 2006). As MWL, assim como outros parâmetros de qualidade, são influenciados pela variedade de sorgo. Bekele et al. (2012) estudaram seis variedades, com $144 \mathrm{~h}$ (seis dias) de germinação, e a perda mais alta $(27,56 \%)$ foi verificada com a variedade Meko, e a perda mais baixa (19,91\%), com a variedade Teshale. Igualmente, Nnamchi et al. (2014) reportaram que, das 10 variedades avaliadas, a maior perda $(37,74 \%)$ ocorreu com a variedade White Kaura e a menor (13,77\%), com a variedade Boboje. Na maltagem, minimizar as perdas e conseguir a modificação necessária dos grãos são metas essenciais para o bom desenvolvimento do processo.

\subsubsection{Poder diastático}

Conforme Novellie (1959), o poder diastático é a capacidade de ambas as enzimas, $\alpha$ e $\beta$-amilase, de hidrolisar o amido em açúcares menores. Graus Lintner $\left({ }^{\circ} \mathrm{L}\right)$ e unidades diastáticas de sorgo (SDU), unidades usadas para expressar o DP, são definidos como a quantidade de atividade enzimática, sob condições determinadas, em dependência do método usado. O autor acrescentou que um grau Lintner é equivalente a meio SDU $\mathrm{g}^{-1}$. É recomendado que o DP do sorgo esteja entre 60 e $80 \mathrm{SDU} \mathrm{g}^{-1}\left(30^{\circ} \mathrm{L}\right.$ e $40^{\circ} \mathrm{L}$ ) (NOVELLIE, 1962b), e que seja de $80^{\circ} \mathrm{L}$ para a cevada (PALMER et al., 1989). O malte de sorgo tem baixo DP, quando comparado ao DP do malte de cevada; assim, o DP é uns dos parâmetros de qualidade mais importantes. O uso de compostos químicos, em baixa concentração na imersão, tais como álcalis, pode melhorar o poder diastático. Por exemplo, é recomendado o uso de até $0,3 \%$ de $\mathrm{Ca}(\mathrm{OH})_{2}, \mathrm{KOH}$ ou $\mathrm{NaOH}$ (DEWAR et al., 1997). Por outro lado, Lefyedi e Taylor (2006) reportaram que, além de aumentar o DP, a imersão dos grãos em $0,2 \%$ de $\mathrm{NaOH}$ é um método recomendado para o controle da contaminação bacteriana e fúngica, durante a maltagem. O poder diastático é influenciado pela temperatura e pela quantidade de água absorvida durante a imersão. Ebbah et al. (2015) reportaram que, a $40{ }^{\circ} \mathrm{C}$, a quantidade de água absorvida pela cultivar (Tabela 1), na imersão, contribui para o alto DP $(88,1$ SDU $\left.\mathrm{g}^{-1}\right)$, devido ao fato de se ter criado um ambiente propício para o desenvolvimento das enzimas. Além disso, o tempo de germinação e a variedade também influenciam o DP. Geralmente, o DP aumenta com o aumento do tempo de germinação em até 120 h (DJAMEH et al., 2015; ABUAJAH et al., 2016a). Bekele et al. (2012) chegaram a um tempo de germinação ótimo de 96 h para obter alto 
DP, nas cultivares estudadas (Tabela 1). Nesse tempo, obtiveram valores entre $23,91^{\circ} \mathrm{L}$ e $31,39^{\circ} \mathrm{L}$ para as variedades 76T1\#23 e Gambella 1107, respectivamente. Ogu et al. (2006) obtiveram, com um tempo de germinação maior (120 h), com a cultivar KSV 4, o maior valor de DP (30 ㄴ), similar ao obtido por Bekele et al. (2012).

\subsubsection{Extrato em água fria}

Conforme Ezeogu e Okolo (1999), o extrato em água fria é um dos indicadores úteis da modificação do malte e representa os produtos solúveis da hidrólise enzimática durante o processo de maltagem. Estes produtos são açúcares disponíveis e aminoácidos localizados no endosperma. Acrescentaram que o valor de CWE do malte bem modificado é cerca de $20 \%$. O CWE também mostra uma tendência de aumentar com o aumento do tempo de germinação entre 96 h e 168 h (PATHIRANA et al., 1983) e pode ser melhorado com um tratamento alcalino durante a imersão (OKOLO; EZEOGU, 1996). O CWE, assim como as MWL e o DP, também é influenciado pela variedade. Nnamchi et al. (2014) estudaram a maltagem, realizada com 24 h de imersão, 96 h de germinação e 24 h de secagem a $48^{\circ} \mathrm{C}$, de 10 cultivares de sorgo (Tabela 1), e os valores de CWE variaram entre $14,80 \%$ e 22,50\% nas cultivares ICSV III e SK 5912, respectivamente. Mas, em um estudo anterior feito por Ogu et al. (2006), com $40 \mathrm{~h}$ de imersão, com um período de quatro horas de AR, $120 \mathrm{~h}$ de germinação e $16 \mathrm{~h}$ de secagem a $50^{\circ} \mathrm{C}$, atingiram-se valores mais altos, de $31 \%$ para a cultivar KSV 8 e de $36 \%$ para as restantes cultivares (SK5912, KSV 4 e ICSV 400). No período de AR, os grãos ficam expostos ao ar por um tempo predeterminado, antes de preencher novamente o recipiente com água fresca (DEWAR et al., 1997). Além das características próprias dos grãos, as quais podem ter uma importante influência, isso pode ser devido às condições de maltagem usadas pelos autores citados. Assim, maiores tempos de imersão, com períodos de AR, e de germinação podem aumentar os valores de CWE. Igualmente, Pathirana et al. (1983), entre 48 h e 144 h de germinação, obtiveram valores superiores de CWE com 18 h $(21 \%$ a $32 \%)$ e com 32 h (21\% a 31\%) de imersão, quando comparados com os CWE obtidos com 8 h $(17 \%$ a $28 \%$ ). Do mesmo modo, esses estudos mostram que tempos de imersão, entre 24 h e 40 h, com períodos de AR e tempos de germinação entre 120 h e 168 h, são recomendados para a maltagem de sorgo, em termos de CWE.

\subsubsection{Extrato em água quente}

O extrato em água quente é uma medida da quantidade de materiais solúveis lixiviados em água sob condições de mosturação a $65^{\circ} \mathrm{C}$ (AGU; PALMER, 1998) e é composto, principalmente, por açúcares fermentáveis (KUMAR et al., 2013). O valor mínimo recomendado de
HWE é de 80\%, em peso seco (AALBERS; EERDE, 1986). Bekele et al. (2012) assinalaram que HWE é um dos mais importantes parâmetros de qualidade do malte. Altos níveis de maltose podem ser obtidos a partir do HWE do malte de sorgo. Pathirana et al. (1983) obtiveram, a partir da variedade amarela (IS 2941), 89,3\% de maltose com $18 \mathrm{~h}$ de imersão e 144 h de germinação. No estudo posterior, feito por Okoli et al. (2010), encontraram que a maltose constituiu o principal açúcar fermentável (74\% a 80\% do total) nas cultivares (Tabela 1), entre $120 \mathrm{~h}$ e $144 \mathrm{~h}$ de germinação. O HWE, assim como o PD, também mostra uma tendência de aumentar com o incremento do tempo de germinação em até 144 h e 168 h (HASSANI et al., 2014). Independentemente do tipo de cultivar, geralmente, o HWE aumenta com o aumento do tempo de germinação; contudo, podem ser obtidos valores diferentes de HWE em função da cultivar, mostrando, mais uma vez, a influência desta na qualidade do malte. Por exemplo, com $144 \mathrm{~h}$ de germinação, Bekele et al. (2012) obtiveram o maior teor de HWE $(85,08 \%)$ com a cultivar Meko, seguida pela Teshale $(83,4 \%)$, enquanto o valor mais baixo $(66,5 \%)$ foi observado na Red-Swazi. Bekele et al. (2012) reportaram um período de maltagem ótimo de $96 \mathrm{~h}$ devido ao fato de que o HWE mostrou pouco incremento, depois desse período. Altos valores de HWE podem ser obtidos com o incremento do tempo de imersão, além de períodos de AR e água morna, ao final da etapa. Foram obtidos 310,70 e $303,50^{\circ} \mathrm{L} \mathrm{kg}^{-1}$ para as cultivares ICSV 400 e KSV 8, respectivamente (EZEOGU; OKOLO, 1995). No caso do malte de sorgo, para obter altos teores de HWE, o método de decantação é mais efetivo do que o método de infusão, usado para o malte de cevada.

\subsubsection{Atenuação limite}

A atenuação limite representa a quantidade de açúcares fermentáveis (geralmente glicose, maltose e maltotriose) presente no mosto preparado a partir do malte. O princípio é que esses açúcares fermentáveis são absorvidos pelas leveduras (na fermentação), resultando na produção de $\mathrm{CO}_{2}$ e etanol (EBBAH et al., 2015). Portanto, tanto o HWE como a AAL dependem diretamente das enzimas amilolíticas. São recomendados valores de atenuação limite entre 81\% e 86\% (ZARNKOW et al., 2007). Odibo et al. (2002) obtiveram, com uma temperatura de imersão de $30{ }^{\circ} \mathrm{C}$, melhores resultados de $\mathrm{AAL}, 78 \%$ e $82 \%$, para as duas cultivares nigerianas de sorgo, SK 5912 e Fara fara, respectivamente, se comparados com a faixa de AAL (71,43\% a 78,95\%) obtida por Ebbah et al. (2015) com a cultivar Kadaga, com $30{ }^{\circ} \mathrm{C}$ e $40^{\circ} \mathrm{C}$ na imersão, respectivamente. Isto se deve a uma quantidade maior de açúcares fermentáveis presentes nas cultivares SK 5912 e Fara fara do que na Kadaga. Além disso, se o mosto contém altas concentrações de maltotriose (parcialmente fermentável por algumas cepas de leveduras) e dextrinas, 
resultará em uma atenuação limite inferior. Djameh et al. (2015) reportaram que a AAL da cultivar Chireh foi significativamente influenciada pelo tempo de imersão. A atenuação limite aumentou de 74,24\% até 78,68\% entre 12 h e 22 h de imersão, mas decresceu ligeiramente de $78,21 \%$ para $77,23 \%$, com o incremento da temperatura de secagem de $30{ }^{\circ} \mathrm{C}$ até $40{ }^{\circ} \mathrm{C}$, ficando praticamente constante entre $40^{\circ} \mathrm{C}$ e $50^{\circ} \mathrm{C}$. O maior valor foi atingido com 22 h de imersão e 120 h de germinação.

\subsubsection{Nitrogênio amino livre}

Conforme Knatchbull e Slaughter (1987), o nitrogênio amino livre do mosto representa a maior fonte de nitrogênio assimilável pelas leveduras, na produção de cerveja. Há relatos de que os níveis necessários são entre 100 e $150 \mathrm{mg} \mathrm{L}^{-1}$, para manter satisfatórios o crescimento das leveduras e a fermentação (PIERCE, 1987). Assim como o DP e o HWE, o FAN é influenciado pelo tempo de germinação e pela variedade. Bekele et al. (2012) reportaram que o aumento do tempo de germinação resultou em um consistente incremento nos valores de FAN nas cultivares estudadas (Tabela 1). Com 144 h, foi obtido o mínimo valor (232,73 $\left.\mathrm{mg} \mathrm{L}^{-1}\right)$ do FAN com a cultivar Teshale e o máximo valor $\left(343,29 \mathrm{mg} \mathrm{L}^{-1}\right)$ com a cultivar 76T1\#23. Evans e Taylor (1990) também reportaram o incremento da atividade proteolítica do malte de sorgo com o incremento do tempo de germinação. Os valores reportados por Bekele et al. (2012) são marcantemente superiores àqueles considerados ótimos para a fermentação, devido ao fato de que os valores do FAN foram determinados a partir dos maltes inteiros, ou seja, incluindo as raízes secas, as quais são ricas em FAN. Sob as mesmas condições de maltagem (Tabela 1), foram obtidos por Nnamchi et al. (2014) diferentes valores de FAN com a cultivar SK 5912 (185 mg L-1) e com a cultivar Nafelen (109 mg L-1). Várias razões podem explicar essas diferenças, tais como condições de cultivo e ambientais, e composição dos grãos. Esse fato demonstra que a cultivar tem um papel chave nos parâmetros de qualidade do malte de sorgo.

\subsubsection{Nitrogênio solúvel total e Índice de Kolbach}

Conforme Evans e Taylor (1990), a atividade proteolítica pode ser medida em termos de solubilização do nitrogênio total e do FAN, pela ação das enzimas proteinases e carboxipeptidases, respectivamente. A solubilização das proteínas durante a maltagem, expressa como TSN, está relacionada com o KI. Conforme Hassani et al. (2014), o Índice de Kolbach é a relação porcentual entre nitrogênio solúvel e o nitrogênio total presente nos grãos. São recomendados valores de KI perto de 40\% (JIN et al., 2012). Os grãos de cevada e de sorgo apresentam teores similares de nitrogênio total (AGU; PALMER, 1999; AGU; PALMER, 2013). No entanto, a habilidade para produzir nitrogênio solúvel é diferente em ambos os maltes. Também, outro fato importante é que ambos os cereais mostram alto potencial para o desenvolvimento do DP com o tempo de germinação, mas, com a atividade proteolítica, é diferente. Por exemplo, com similar nitrogênio total (\% seco), 1,63 e 1,60 para a cevada e o sorgo, respectivamente, Agu e Palmer (2013) reportaram que, às $120 \mathrm{~h}$ de germinação, o DP e o nitrogênio solúvel foram $46^{\circ} \mathrm{L}$ e $0,65 \%$ e $32^{\circ} \mathrm{L}$ e $0,45 \%$, para a cevada e o sorgo, respectivamente. Os autores acrescentaram que não há uma relação clara entre o desenvolvimento do DP e o nível de proteólise resultante da liberação de nitrogênio solúvel durante a maltagem dos dois cereais. Seus resultados confirmam que o sorgo tem potencial, em termos de qualidade (DP, HWE e nitrogênio solúvel), para ser usado na fabricação de cerveja, quando se trabalha nas condições ótimas (Tabela 2) de maltagem e usa-se o método de mosturação por decantação em vez do método de infusão. Por outra parte, os resultados de Hassani et al. (2014) mostraram que um maior grau de imersão (umidade ao final da imersão) e um maior tempo de germinação levaram a altos Índices de Kolbach (30,30\%). Portanto, estes estudos mostram que as limitações do sorgo na produção de cerveja podem ser minimizadas com a otimização do processo de maltagem e a etapa de mosturação. Na Tabela 1, são apresentados alguns dos fatores que têm otimizado parâmetros de qualidade do malte de sorgo.

\section{Mosturação}

Os objetivos da mosturação são de formar e de extrair, em solução, açúcares fermentáveis, aminoácidos, vitaminas etc., a partir do malte. Dentre as principais enzimas envolvidas na hidrólise do amido estão $\alpha$ e $\beta$-amilase, dextrinase limite e $\alpha$-glicosidase. Além disso, são muito importantes as enzimas proteolíticas, as quais hidrolisam as proteínas. A eficiência da mosturação depende do desenvolvimento dessas enzimas, na maltagem, e do regime de temperatura e tempo usado na mosturação. Conforme Brosnan et al. (1992), a $\alpha$-amilase catalisa a hidrólise aleatória das ligações glicosídicas $\alpha(1,4)$ internas, liberando pequenos oligossacarídeos e glicose. Já a $\beta$-amilase atua na penúltima ligação glicosídica $(1,4)$, na extremidade não redutora, liberando maltose (MA et al., 2000). Ambas as enzimas não hidrolisam as ligações $\alpha(1,6)$, mas a dextrinase limite realiza essa função (MANNERS et al., 1970). Outra enzima que catalisa a hidrólise das ligações $\alpha(1,4)$ é a $\alpha$-glicosidase, liberando glicose a partir das ligações terminais não redutoras (TAYLOR; DEWAR, 1994). Por outra parte, as enzimas proteolíticas (ou proteases) catalisam a quebra de grandes moléculas de proteínas, hidrolisando ligações peptídicas, liberando pequenos fragmentos de proteínas ou aminoácidos livres como produtos (OGBONNA, 2009). A Tabela 3 apresenta valores de $\mathrm{pH}$ e de temperatura ótimos para cada uma destas enzimas, as quais são ativadas na 
Utilização do malte de sorgo na produção de cerveja: revisão bibliográfica

Rodríguez, Y. B. et al.

Tabela 3. Parâmetros ótimos das principais enzimas envolvidas na hidrólise do amido e das proteínas.

\begin{tabular}{|c|c|c|c|}
\hline Enzima & pH ótimo & Temperatura ótima $\left({ }^{\circ} \mathbf{C}\right)$ & Referência \\
\hline$\alpha$-amilase & 5.0 & 70 & \multirow{2}{*}{ (EL-NOUR; YAGOUB, 2010) } \\
\hline$\beta$-amilase & 5.5 & 50 & \\
\hline Dextrinase limite & $5.0-5.4$ & 60 & (HARDIE et al., 1976) \\
\hline$\alpha$-glicosidase & $3.75-4.0$ & 60 & (TAYLOR; DEWAR, 1994) \\
\hline Enzimas proteolíticas (proteases) & $5.6-6.0$ & 50 & (OGBONNA, 2009) \\
\hline
\end{tabular}

mosturação. A mosturação pode ser feita por infusão ou por decantação. Um estudo feito por Ndubisi et al. (2016) sobre a mosturação por infusão (temperatura constante de $65^{\circ} \mathrm{C}$ ) de uma variedade amarela de sorgo a $65^{\circ} \mathrm{C}$ resultou em baixos valores de HWE (100 $\left.{ }^{\circ} \mathrm{kg}^{-1}\right)$. Já com enzimas comerciais, tanto a $65^{\circ} \mathrm{C}$ como a $85^{\circ} \mathrm{C}$, os valores de HWE melhoraram, indicando a temperatura de $65^{\circ} \mathrm{C}$ como a mais adequada para o $\mathrm{HWE}, 300^{\circ} \mathrm{L} \mathrm{kg}^{-1}$. Alternativamente, para atingir o mesmo objetivo, a mosturação pode ser feita a $85^{\circ} \mathrm{C}$ sem a adição de enzimas comerciais, para reduzir custos. A mosturação por decantação (decocção) é amplamente aplicada na África (LYUMUGABE et al., 2015). A decantação usada por Agu e Palmer (2013) consistiu na obtenção do extrato a $30{ }^{\circ} \mathrm{C}$ por 30 min, decantação do extrato enzimático, fervura do extrato residual até $100^{\circ} \mathrm{C}$, para gelatinizar o amido, e, quando frio, misturar ambos os mostos para realizar a mosturação a $65^{\circ} \mathrm{C}$ por $1 \mathrm{~h}$. Com esse método, os autores atingiram um valor de HWE do malte de sorgo (ver Tabela 1) superior ao valor de HWE do malte de cevada (323 ${ }^{\circ} \mathrm{kg}^{-1}$ ), quando foi usado o método de infusão $\left(65^{\circ} \mathrm{C}\right.$ por $\left.1 \mathrm{~h}\right)$. Por outra parte, a mosturação por decantação produz mostos com maior quantidade de açúcares fermentáveis, quando comparada à mosturação por infusão. Por exemplo, Archibong et al. (2015) estudaram ambos os métodos, usando enzimas a partir de Aspergillus sydowii, com duas variedades de sorgo. Quando usado o método de infusão, as concentrações de glicose, maltose, maltotriose e dextrina foram 26,41 , 13,33, 8,24 e 9,26 $\mathrm{mg} \mathrm{mL}^{-1}$ com enzimas e 25,91, 12,7, 4,72 e $11,55 \mathrm{mg} \mathrm{mL}^{-1}$ sem enzimas, respectivamente, para a variedade SK5912. Já os resultados foram inferiores no mosto da variedade CSR01, em que as concentrações de glicose, maltose, maltotriose e dextrinas foram 19,59, 3,7, 4,07 e 4,33 $\mathrm{mg} \mathrm{mL}^{-1}$ com enzimas e 15,18, 3,65, 3,91 e $5 \mathrm{mg}$ $\mathrm{mL}^{-1}$ sem enzimas, respectivamente. No entanto, quando feita a mosturação por decantação, os resultados foram melhores. Para a variedade SK5912, as concentrações de glicose, maltose, maltotriose e dextrina foram 37,71 , 25,77, 17,48 e 9,33 $\mathrm{mg} \mathrm{mL}^{-1}$ com enzimas e 21,27, 10,91, 8,8 e $14,45 \mathrm{mg} \mathrm{L}^{-1}$ sem enzimas, respectivamente.

Já para a variedade CSR01, os valores foram 31,06, 15,57, 8,28 e 4,32 $\mathrm{mg} \mathrm{mL}^{-1}$ com enzimas e 23,16, 12,38, 8,19 e 4,35 $\mathrm{mg} \mathrm{mL}^{-1}$ sem enzimas. Como observado na maltagem, a variedade também influencia na etapa de mosturação. As diferenças observadas por esses autores nos açúcares fermentáveis podem ser decorrentes do nível de enzimas hidrolíticas de cada variedade. Deste modo, quando se trabalha com malte de sorgo, a mosturação por decantação é mais adequada do que a mosturação por infusão, constituído assim um método de otimização.

\section{Considerações finais}

O conhecimento dos fatores que influenciam o processo de maltagem e a etapa de mosturação permite o entendimento necessário do processo de produção de bebidas fermentadas com sorgo. Os fatores que otimizam os parâmetros de qualidade do malte, tais como tempo e temperatura de imersão, de germinação e de secagem, assim como o pH e a temperatura das enzimas, e o apropriado método de mosturação, resumidos na presente revisão, fornecem informações que podem apoiar a escolha do malte de sorgo como substituto do malte de cevada. Considerando-se as vantagens do sorgo, dos pontos de vista agronômico e alimentar, os estudos já existentes fornecem informações capazes de sustentar a utilização do seu malte para esse fim.

\section{Agradecimentos}

Os autores agradecem ao Conselho Nacional de Desenvolvimento Científico e Tecnológico (CNPq, Processo $n^{\circ}$ 870130/2011-0), pelo apoio financeiro e por concessão de bolsa a Yanet Boffill Rodríguez.

\section{Referências}

AALBERS, V. J.; EERDE, P. Centenary review evaluation of malt quality. Journal of the Institute of Brewing, v. 92, n. 5, p. 420-425, 1986. http://dx.doi.org/10.1002/j.2050-0416.1986.tb04434.x.

ABUAJAH, C. I.; OGBONNA, A. C.; ONWUKA, N. U.; UMOREN, P. E.; OJUKWU, M. Effect of varietal differences and germination period on some malting and brewing potentials of new improved sorghum varieties (SAMSORG17, SAMSORG14, and SAMSORG40) from Nigeria. International Food Research Journal, v. 23, n. 4, p. 1600-1608, 2016a. Disponível em: <http://www.ifrj.upm.edu. my/23\%20(04)\%202016/(33).pdf>. Acesso em: 19 nov. 2015.

ABUAJAH, C. I.; OGBONNA, A. C.; SONDE, C. U.; OFFIA-OLUA, B.; OWHOEKE, E. Variety and germination time effect on total $\beta$-glucan, water-insoluble $\beta$-glucan, water-soluble $\beta$-glucan components and $\beta$-glucanase levels in improved sorghum varieties 
Utilização do malte de sorgo na produção de cerveja: revisão bibliográfica

Rodríguez, Y. B. et al.

SK5912, KSV8 and ICSV400 before and after malting and their relationships to wort viscosity. Journal of the Institute of Brewing, v. 122, n. 1, p. 93-101, 2016b. http://dx.doi.org/10.1002/jib.300.

AGU, R. C.; PALMER, G. H. Enzymic breakdown of endosperm proteins of sorghum at different malting temperatures. Journal of the Institute of Brewing, v. 102, n. 6, p. 415-418, 1996. http:// dx.doi.org/10.1002/j.2050-0416.1996.tb00924.x

AGU, R. C.; PALMER, G. H. The effect of temperature on the modification of sorghum and barley during malting. Process Biochemistry, v. 32, n. 6, p. 501-507, 1997. http://dx.doi. org/10.1016/S0032-9592(97)00002-2.

AGU, R. C.; PALMER, G. H. A reassessment of sorghum for lager-beer brewing. Bioresource Technology, v. 66, n. 3, p. 253-261, 1998. http://dx.doi.org/10.1016/S0960-8524(98)00047-9.

AGU, R. C.; PALMER, G. H. Comparative development of soluble nitrogen in the malts of barley and sorghum. Process Biochemistry, v. 35, n. 5, p. 497-502, 1999. http://dx.doi. org/10.1016/S0032-9592(99)00092-8.

AGU, R. C.; PALMER, G. H. Evaluation of the potentials of millet, sorghum and barley with similar nitrogen contents malted at their optimum germination temperatures for use in brewing. Journal of the Institute of Brewing, v. 119, n. 4, p. 258-264, 2013. http:// dx.doi.org/10.1002/jib.91.

AISEN, A. O.; MUTS, G. C. J. Micro-scale malting and brewing studies of some sorghum varieties. Journal of the Institute of Brewing, v. 93, n. 4, p. 328-331, 1987. http://dx. doi. org/10.1002/j.2050-0416.1987.tb04513.x.

ARCHIBONG, E. J.; ONUORA, V. C.; USOEGBU, O.; EZEMBA, C. C. Mashing studies of two (2) improved sorghum varieties (SK5912 and CSR01) using crude enzyme extract from Aspergillus sydowii. World Journal of Pharmacy and Pharmaceutical Sciences, v. 4, p. 113-125, 2015.

ARENDT, E. K.; SCHNITZENBAUMER, B. Brewing with up to $40 \%$ unmalted oats (Avena sativa) and sorghum (Sorghum bicolor): a review. Journal of the Institute of Brewing, v. 120, p. 315-330, 2014.

BEKELE, A.; BULTOSA, G.; BELETE, K. The effect of germination time on malt quality of six sorghum (Sorghum bicolor) varieties grown at Melkassa, Ethiopia. Journal of the Institute of Brewing, v. 118, n. 1, p. 76-81, 2012. http://dx.doi.org/10.1002/jib. 19.

BROSNAN, M. P.; KELLY, C. T.; FOGARTY, W. M. Investigation of the mechanisms of irreversible thermoinactivation of Bacillus stearothermophilus $\alpha$-amylase. European Journal of Biochemistry, v. 203, n. 1-2, p. 225-231, 1992. http://dx.doi. org/10.1111/j.1432-1033.1992.tb19850.x. PMid:1730228.

CLAVER, I. P.; ZHANG, H.; LI, Q.; ZHOU, H.; ZHU, K. Optimized conditions of steeping and germination and their effect on sorghum [Sorghum bicolor (L.) Moench] composition. Pakistan Journal of Nutrition, v. 9, n. 7, p. 686-695, 2010. http://dx.doi. org/10.3923/pjn.2010.686.695.
DEWAR, J.; TAYLOR, J. R. N.; BERJAK, P. Determination of improved steeping conditions for Sorghum malting. Journal of Cereal Science, v. 26, n. 1, p. 129-136, 1997. http://dx.doi. org/10.1006/jcrs. 1996.0101

DJAMEH, C.; SAALIA, F. K.; SINAYOBYE, E.; BUDU, A.; ESSILFIE, G.; MENSAH-BROWN, H.; SEFA-DEDEH, S. Optimization of the sorghum malting process for pito production in Ghana. Journal of the Institute of Brewing, v. 121, n. 1, p. 106-112, 2015. http:// dx.doi.org/10.1002/jib.191.

DUFOUR, J. P.; MÉLOTTE, L.; SREBRNIK, S. Sorghum malts for the production of lager beer. Journal of the American Society of Brewing Chemists, v. 50, n. 3, p. 110-119, 1992. http://dx.doi. org/10.1094/ASBCJ-50-0110.

EBBAH, L.; LARYEA, D.; BARIMAH, J.; DJAMEH, C. Effect of steeping temperature on the quality of malt and Pito (an indigenous Ghanaian drink). Journal of the Institute of Brewing, v. 121, n. 4, p. 518-523, 2015. http://dx.doi.org/10.1002/jib.265.

EL-NOUR, M. E. M.; YAGOUB, S. O. Partial purification and characterization of $\alpha$ and $\beta$-Amylase isolated from Sorghum bicolor c.v. (Feterita) malt. Journal of Applied Sciences, 2010, 10: 1314-1319.

EVANS, D. J.; TAYLOR, J. R. N. Extraction and assay of proteolytic activities in sorghum malt. Journal of the Institute of Brewing, v. 96, n. 4, p. 201-207, 1990. http://dx.doi.org/10.1002/j.2050-0416.1990. tb01028.x.

EZEOGU, L. I.; OKOLO, B. N. Effects of air rest periods on malting sorghum response to final warm water steep. Journal of the Institute of Brewing, v. 101, n. 1, p. 39-45, 1995. http:// dx.doi.org/10.1002/j.2050-0416.1995.tb00848.x.

EZEOGU, L. I.; OKOLO, B. N. Relationship between some sorghum malt quality characteristics and nature of alkaline steep. Journal of the Institute of Brewing, v. 105, n. 1, p. 49-54, 1999. http:// dx.doi.org/10.1002/j.2050-0416.1999.tb00005.x.

FASANO, A.; CATASSI, C. Current approaches to diagnosis and treatment of celiac disease: an evolving spectrum. Gastroenterology, v. 120, n. 3, p. 636-651, 2001. http://dx.doi. org/10.1053/gast.2001.22123. PMid:11179241.

FOOD AND AGRICULTURE ORGANIZATION - FAO. Produces prices - annual. Rome: FAO, 2015. Disponível em: <http://www. fao.org/faostat/en/\#data/PP>

GOMES, L. O. F.; SANTIAGO, R. A. C.; KOAKUZU, S. N.; BASSINELLO, P. Z. Estabilidade microbiológica e físico-química de misturas para bolo sem glúten e qualidade dos bolos prontos para consumo. Brazilian Journal of Food Technology, v. 17, n. 4, p. 283-295, 2014. http://dx.doi.org/10.1590/1981-6723.0914.

HARDIE, D. G.; MANNERS, D. J.; YELLOWLEES, D. The limit dextrinase from malted sorghum (Sorghum vulgare). Carbohydrate Research, v. 50, n. 1, p. 75-85, 1976. http://dx.doi.org/10.1016/ S0008-6215(00)84084-7. PMid:975121. 
Utilização do malte de sorgo na produção de cerveja: revisão bibliográfica

Rodríguez, Y. B. et al.

HASSANI, A.; ZARNKOW, M.; BECKER, T. Influence of malting conditions on sorghum (Sorghum bicolor (L.) Moench) as a raw material for fermented beverages. Food Science \& Technology International, v. 20, n. 6, p. 453-463, 2014. http://dx.doi. org/10.1177/1082013213490710. PMid:23751551

JIN, Y.; DU, J.; ZHANG, K.; XIE, L.; LI, P. Relationship between Kolbach index and other quality parameters of wheat malt. Journal of the Institute of Brewing, v. 118, n. 1, p. 57-62, 2012. http:// dx.doi.org/10.1002/jib. 15 .

KNATCHBULL, F. B.; SLAUGHTER, C. The effect of low $\mathrm{CO}_{2}$ pressures on the absorption of amino acids and production of flavour-active volatiles by yeast. Journal of the Institute of Brewing, v. 93, n. 5, p. 420-424, 1987. http://dx.doi. org/10.1002/j.2050-0416.1987.tb04530.x.

KUMAR, D.; KUMAR, V.; VERMA, R. P. S.; KHARUB, A. S.; SHARMA, I. Quality parameter requirement and standards for malt barley-a review. Agricultural Reviews, v. 34, n. 4, p. 313-317, 2013. http://dx.doi.org/10.5958/j.0976-0741.34.4.018.

LEFYEDI, M. L.; TAYLOR, J. R. N. Effect of dilute alkaline steeping on the microbial contamination, toxicity and diastatic power of sorghum malt. Journal of the Institute of Brewing, v. 112, n. 2, p. 108-116, 2006. http://dx.doi.org/10.1002/j.2050-0416.2006. tb00240.x.

LYUMUGABE, F.; GROS, J.; BAJYANA SO, E.; THONART, $P$. Sorghum beer brewing using eleusine coracana "Finger Millet" to improve the saccharification. American Journal of Food Technology, v. 10, n. 4, p. 167-175, 2015. http://dx.doi. org/10.3923/ajtt.2015.167.175.

MA, Y.; STEWART, D. C.; EGLINTON, J. K.; LOGUE, S. J.; LANGRIDGE, P.; EVANS, D. E. Comparative enzyme kinetics of two allelic forms of Barley (Hordeum vulgare L.) beta-amylase. Journal of Cereal Science, v. 31, n. 3, p. 335-344, 2000. http:// dx.doi.org/10.1006/jcrs.2000.0303.

MANNERS, D. J.; MARSHALL, J. J.; YELLOWLEES, D. The specificity of cereal limit dextrinases. The Biochemical Journal, V. 116, n. 3, p. 539-541, 1970. http://dx.doi.org/10.1042/bj1160539. PMid:5435695

MARIANI, M.; OLIVEIRA, V. R.; FACCIN, R.; RIOS, A. O.; VENZKE, J. G. Elaboração e avaliação de biscoitos sem glúten a partir de farelo de arroz e farinhas de arroz e de soja. Brazilian Journal of Food Technology, v. 18, n. 1, p. 70-78, 2015. http://dx.doi. org/10.1590/1981-6723.6514

MEZGEBE, A. G.; ABEGAZ, K.; TAYLOR, J. R. N. Relationship between waxy (high amylopectin) and high protein digestibility traits in sorghum and malting quality. Journal of Cereal Science, v. 79, p. 319-327, 2018. http://dx.doi.org/10.1016/j.jcs.2017.11.015.

MOKHAWA, G.; KERAPELETSWE-KRUGER, C. K.; EZEOGU, L. I. Electrophoretic analysis of malting degradability of major sorghum reserve proteins. Journal of Cereal Science, v. 58, n. 1, p. 191-199, 2013. http://dx.doi.org/10.1016/j.jcs.2013.05.008.
NDUBISI, C. F.; OKAFOR, E. T.; AMADI, O. C.; NWAGU, T. N.; OKOLO, B. N.; MONEKE, A. N.; ODIBO, F. J. C.; OKORO, P. M.; AGU, R. C. Effect of malting time, mashing temperature and added commercial enzymes on extract recovery from a Nigerian malted yellow sorghum variety. Journal of the Institute of Brewing, $v$. 122, n. 1, p. 156-161, 2016. http://dx.doi.org/10.1002/jib.307.

NNAMCHI, C. I.; OKOLO, B. N.; MONEKE, A. N. Grain and malt quality properties of some improved Nigerian sorghum varieties. Journal of the Institute of Brewing, v. 120, p. 353-359, 2014.

NOVELLIE, L. Kaffircorn malting and brewing studies. III. Determination of amylases in kaffircorn malts. Journal of the Science of Food and Agriculture, v. 10, n. 8, p. 441-449, 1959. http://dx.doi.org/10.1002/jsfa.2740100808.

NOVELLIE, L. Kaffir corn malting and brewing studies XII. Effect of malting conditions on malting losses and total amylases activity. Journal of the Science of Food and Agriculture, v. 13, n. 2, p. 121-123, 1962a. http://dx.doi.org/10.1002/jsfa.2740130211.

NOVELLIE, L. Kaffircorn malting and brewing studies. XIII. Variation of diastatic power with variety, season, maturity and age of grain. Journal of the Science of Food and Agriculture, v. 13, n. 2, p. 124-126, 1962b. http://dx.doi.org/10.1002/jsfa.2740130212.

ODIBO, F. J. C.; NWANKWO, L. N.; AGU, R. C. Production of malt extract and beer from Nigerian sorghum varieties. Process Biochemistry, v. 37, n. 8, p. 851-855, 2002. http://dx.doi. org/10.1016/S0032-9592(01)00286-2.

OGBONNA, A. C. Proteolytic enzymes and protein modification in malting sorghum: a review. Master Brewers Association of the Americas Technical Quarterly, v. 10, n. 1, p. 1-6, 2009. http://dx.doi.org/10.1094/TQ-46-3-0714-01.

OGBONNA, A. C. Current developments in malting and brewing trials with sorghum in Nigeria: a review. Journal of the Institute of Brewing, v. 117, n. 3, p. 394-400, 2011. http://dx. doi. org/10.1002/j.2050-0416.2011.tb00485.x

OGU, E. O.; ODIBO, F. J. C.; AGU, R. C.; PALMER, G. H. Quality assessment of different sorghum varieties for their brewing potential. Journal of the Institute of Brewing, v. 112, n. 2, p. 117-121, 2006. http://dx.doi.org/10.1002/j.2050-0416.2006.tb00241.x.

OKOLI, E. V.; OKOLO, B. N.; MONEKE, A. N.; IRE, F. S. Effects of cultivar an germination time on amynolitic potential, extract yield and wort fermenting properties of malting sorgum. Asian Journal of Biotechnology, v. 2, n. 1, p. 14-26, 2010. http:// dx.doi.org/10.3923/ajbkr.2010.14.26.

OKOLO, B. N.; EZEOGU, L. I. Enhancement of amylolytic potential of sorghum malts by alkaline steep treatment. Journal of the Institute of Brewing, v. 102, n. 2, p. 79-85, 1996. http://dx.doi. org/10.1002/j.2050-0416.1996.tb00897.x.

OWUAMA, C. I. Sorghum: a cereal with lager beer brewing potential. World Journal of Microbiology \& Biotechnology, v. 13, n. 3, p. 253-260, 1997. http://dx.doi.org/10.1023/A:1018566503879. 
Utilização do malte de sorgo na produção de cerveja: revisão bibliográfica

Rodríguez, Y. B. et al.

OWUAMA, C. I.; OKAFOR, N. Studies on mashing methods for beer brewing with sorghum. MIRCEN Journal of Applied Microbiology and Biotechnology, v. 3, n. 3, p. 243-253, 1987. http://dx.doi.org/10.1007/BF00933578.

PALMER, G. H.; ETOKAKPAN, O. U.; IGYOR, M. A. Sorghum as brewing material. MIRCEN Journal of Applied Microbiology and Biotechnology, v. 5, n. 3, p. 265-275, 1989. http://dx.doi. org/10.1007/BF01741756.

PATHIRANA, R. A.; SIVAYOGASUNDARAM, K.; JAYATISSA, P. M. Optimisation of conditions for malting of sorghum. Journal of Food Science and Technology, v. 20, n. 3, p. 108-111, 1983.

PIERCE, J. S. The role of nitrogen in brewing. Journal of the Institute of Brewing, v. 93, n. 5, p. 378-381, 1987. http://dx.doi. org/10.1002/j.2050-0416.1987.tb04520.x.

SILVA, C.; BRITO, J.; SOUZA, M. Z.; SILVEIRA, A.; LUVIELMO, M. M.; SALAS, M. M. Adição de colágeno em pão sem glúten elaborado com farinha de arroz. Brazilian Journal of Food Technology, v. 20, p. 2-10, 2017.

TAYLOR, B. J. R. N.; DEWAR, J. Role of alpha-glucosidase in the fermentable sugar composition of sorghum malt mashes. Journal of the Institute of Brewing, v. 100, n. 6, p. 417-419, 1994. http://dx.doi.org/10.1002/j.2050-0416.1994.tb00840.x.
TAYLOR, J. R. N.; ROBBINS, D. J. Factors influencing betaamylase activity in sorghum malt. Journal of the Institute of Brewing, v. 99, n. 5, p. 413-416, 1993. http://dx.doi. org/10.1002/j.2050-0416.1993.tb01181.x.

TOKPOHOZIN, S. E.; FISCHER, S.; BECKER, T. Assessment of malting and mash bio-acidification on the turnover of sorghum cyanogenic glucoside and protein hydrolysis improvement. Lebensmittel-Wissenschaft + Technologie, v. 90, p. 303-309, 2018. http://dx.doi.org/10.1016/j.Iwt.2017.12.043.

URIYO, M.; EIGEL, W. E. Duration of kilning treatment on $\alpha$-amylase, $\beta$-amylase and endo- $(1,3)(1,4)-\beta$-D- glucanase activity of malted sorghum (Sorghum bicolor). Process Biochemistry, v. 35 , n. 5, p. 433-436, 1999. http://dx.doi.org/10.1016/S00329592(99)00071-0

ZARNKOW, M.; KESSLER, M.; BURBERG, F.; BACK, W.; ARENDT, E. K.; KREISZ, S. KEßLER, M.; BURBERG, F.; BACK, W.; ARENDT, E. K.; KREISZ, S. The use of response surface methodology to optimise malting conditions of proso millet (Panicum miliaceum L.) as a raw material for gluten-free foods. Journal of the Institute of Brewing, v. 113, n. 3, p. 280-292, 2007. http://dx.doi. org/10.1002/j.2050-0416.2007.tb00288.x. 